\title{
Einleitendes zu Teil III
}

\begin{abstract}
The scientist has a lot of experience with ignorance and doubt and uncertainty, and this experience is of very great importance, I think. When a scientist doesn't know the answer to a problem, he is ignorant. When he has a hunch as to what the result is, he is uncertain. And when he is pretty darn sure of what the result is going to be, he is still in some doubt. We have found it of paramount importance that in order to progress we must recognize our ignorance and leave room for doubt. Scientific knowledge is a body of statements of varying degrees of certainty-some most unsure, some nearly sure, but none absolutely certain.
\end{abstract}

Feynman (1955: 14)

Feynmans nuancierte und eloquente Betrachtung wissenschaftlicher Sicherheit stellt im Kern eine Mahnung dar, niemals von einer Absolutheit erreichten Wissens auszugehen, jedoch kann darin auch eine nennenswerte Begründung des wissenschaftlichen Strebens in Bereichen, die methodisch und konzeptuell nur schwer erschlossen werden können, gefunden werden. Obgleich beispielsweise die Wirtschafts-, Sozial- und Sprachwissenschaften damit zu kämpfen haben, dass sie nicht dieselbe mathematische Präzision vorweisen können wie die Physik und die Chemie, so stellen sie dennoch bedeutsame Unternehmungen der menschlichen Wissensfindung dar. Damit stehen einschlägige Wissenschaftler vor der Verantwortung, auch unter erschwerten Bedingungen eine so adäquate Methodologie zu finden wie eben möglich - und auch wenn Ergebnisse nur graduell gesichert sind, so sind dennoch möglichst gute Ergebnisse anzustreben und schließlich mit angemessener Vorsicht vorzubringen.

In Folge dieser Ausführungen zeigt sich die Innovation und der Mehrwert des bereits vorgestellten Methodenkatalogs schließlich in voller Pracht, denn er erlaubt eine methodengeleitete Rezeption der einschlägigen Forschung in einer Weise, die innerhalb der einschlägigen Forschung aktuell nicht üblich ist. Mithin wird das Forschungsfeld durch die Etablierung desselben produktiv bereichert, indem erstmals ein gerichtet anleitendes methodisches Programm zur Bewertung der einschlägigen interdisziplinären Forschung zur Verfügung gestellt wird. Dabei steht eine Demonstration der Anwendbarkeit desselben an gegebener Stelle freilich noch aus. In anderen Worten erreicht die vorliegende Arbeit mit ihren einführenden Abschnitten, der Vorstellung jenes methodischen Programms und der Übersicht zum Inventar der Sprachursprungsforschung ihre thematische Kulmination nun mit dem vorliegenden Teil III, welcher dabei mehrere Funktionen erfüllt.

Erstens zeigt sich anhand der nachfolgend vorgestellten Ergebnisse die Leistungsfähigkeit des bereits diskutierten Methodenkatalogs, welcher mit der 
Intention konstruiert wurde, metawissenschaftliche Sensibilitäten in den Forschungsbereich der Sprachursprungsforschung einzubringen. Die dabei eröffneten Kapitel sind exemplarischer Natur und führen in verschiedentlicher Hinsicht zu neuartigen, thematisch einschlägig relevanten Ergebnissen, womit die Anwendbarkeit des Methodenkatalogs demonstriert werden soll. Dabei verbleibt $\mathrm{zu}$ hoffen, dass sich ein entsprechendes metawissenschaftliches Bewusstsein - sei es mit oder ohne Beitrag durch die vorliegende Arbeit - zunehmend innerhalb der einschlägigen Forschung zu verbreiten vermag.

Zweitens entstehen im Kontext der nachfolgenden Ausführungen gut nachvollziehbare Aufbereitungen zu Instanzen innerhalb der Sprachursprungsforschung, in welchen interdisziplinäre und methodische Probleme des Forschungsfeldes verdeutlicht werden können. Derartige Schwierigkeiten konnten bisher lediglich erwähnt bzw. knapp und ohne Bezug zu konkreten Beispielen skizziert werden. Nachfolgend wird praxisnah zu demonstrieren sein, in welcher Weise jene Probleme tatsächlich in der einschlägigen Literatur vorkommen und inwieweit der vorliegende Methodenkatalog hilft, diese zu erkennen und zu beheben. Hieraus ergibt sich ein zusätzlicher wissenschaftlicher Mehrwert auch dadurch, dass ein solches Aufzeigen problematischer Vorgehensweisen bei Rezipienten dieser Arbeit eine potenzielle Besserung des einschlägigen methodischen Verständnisses eröffnet.

Drittens ergibt sich freilich durch die Analyseergebnisse an sich bereits ein wissenschaftlicher Mehrwert. Innerhalb der einschlägigen Literatur finden sich weitläufig Argumente, welche nicht mit ausreichender Sorgfalt konstruiert wurden und daher $\mathrm{zu}$ problematischen Schlussfolgerungen führen. Daraus entsteht eine weitläufig in sich zerstrittene Forschungslandschaft, in der auch nur provisorische Antworten kaum erreichbar zu sein erscheinen. Die nachfolgenden Erläuterungen stellen dieser undurchsichtigen Situation und den problematischen Positionen angemessene, wenn auch provisorische, Antworten entgegen. In diesem Zuge zeigt sich die vorliegende Arbeit als nicht nur methodisch, sondern auch fachlich produktiv.

Zuletzt erlaubt der dabei aufgespannte Gesamtkontext die Integration potenziell wegweisender Konzepte und Forschungsfelder. Wie zuvor bereits angesprochen handelt es sich dabei um Metakognition als relevantes Merkmal in der evolutionären Selektion hin zu modern-menschlicher Kognition und Sprachbefähigung, um Emergenz als ein Modus der Innovation bzw. Revolution im Verlauf der menschlichen evolutionären Entwicklung und um den Erstspracherwerb als ein fruchtbares Fenster in den Sprachursprung. Dieses Vorgehen erweitert den fachlichen Nutzen dieser Arbeit für die einschlägige Forschung zusätzlich und rundet den Wert des vorliegenden Projekts ab. 